\title{
Does superficial fat affect metabolite concentrations determined by MR spectroscopy with water referencing?
}

\author{
S.P. Kyathanahally ${ }^{1,2}$, N.D. Fichtner ${ }^{1,2,3}$, V. Adalid ${ }^{1,2}$, R. Kreis ${ }^{1}$ \\ 1. Department of Clinical Research and Institute of Diagnostic, Interventional and Pediatric Radiology, \\ University of Bern, Bern, Switzerland \\ 2. Graduate School for Cellular and Biomedical Sciences; University of Bern, Bern, Switzerland \\ 3. Institute for Biomedical Engineering, University and ETH Zurich, Zurich, Switzerland
}

Running Head: Potential effects of superficial fat on metabolite concentrations

Manuscript Word Count: 2254

\section{Corresponding author:}

Prof. Dr. sc. nat. Roland Kreis

University Bern,

Inselspital, P.O. Box 35,

CH-3010 Bern, Switzerland

Tel: +41-31-632 8174, Fax: +41-31-6320580

Email: roland.kreis@insel.ch 


\begin{abstract}
It has recently been reported in this journal that local fat depots produce a sizable frequencydependent signal attenuation in MR spectroscopy of the brain. If of a general nature, this effect would question the use of internal reference signals for quantification of MR spectroscopy and the quantitative use of MR spectroscopy as a whole. Here, it was attempted to verify this effect and pinpoint the potential causes by acquiring data with various acquisition settings, incl. two field strengths, two MR scanners from different vendors, different water suppression sequences, RF coils, localization sequences, echo times, and lipid/metabolite phantoms. With all settings tested, the reported effect could not be reproduced and it is concluded that water referencing and quantitative MR spectroscopy per se remain valid tools under common acquisition conditions.
\end{abstract}

Keywords: MR spectroscopy; brain; quantification; fat; 


\section{List of Abbreviations}

Ace, Acetate

BMI, Body mass index

Cho, Choline

$\mathrm{Cr}$, Creatine

Gly, Glycine

HLSVD, Hankel-Lanczos singular value decomposition

jMRUI, Java version of the magnetic resonance user interface

MC, Metabolite cycling for water suppression

MC+WS, Metabolite cycling plus standard water suppression

NAA, N-acetylaspartate

nWS, Non-water-suppressed

PA, Phased array head coil

PRESS, Point-resolved spectroscopy

QUEST, Quantitation based on quantum estimation

RF, Radio frequency

ROI, Region of interest

SD, Standard deviation

SNR, Signal-to-noise ratio

STEAM, Stimulated echo acquisition mode

SVS, Single voxel spectroscopy

TxRx, Transmit/receive head coil

VESPA, Versatile simulation- pulses and analysis

WS, Water-suppressed 


\section{INTRODUCTION:}

Magnetic resonance spectroscopy (MRS) is known to be a non-invasive tool that allows the determination of tissue content of certain metabolites in vivo - either in relative terms comparing one metabolite to another or in absolute terms if appropriate referencing information is available. There is general agreement that the signal of co-localized water is a clinically robust means to enable semi-absolute quantification (1). The main advantages of using water as an internal reference were supposed to be its equal exposure to complicating factors, such as (i) the exact nature of the pulse profile (ii) the local $\mathrm{B}_{1}{ }^{+}$and $\mathrm{B}_{1}{ }^{-}$amplitudes and field distributions, which depend on the coil geometry and the local arrangement of tissues with differing permittivity and permeability properties, (iii) coil loading, or (iv) non-ideal lineshapes (i.e. $B_{0}$ inhomogeneities). Open issues that are mentioned for water referencing usually refer to inaccurately known tissue water content and relaxation times. However, a recent report (2) on direct, frequency-dependent influences of local lipid depots on MR signal strength has questioned the basis of water referencing and the potential for meaningful quantification using ${ }^{1} \mathrm{H}$ MRS in general.

The background for the investigation of potential influences of the local fat distribution was a dispute in the literature on the reasons for reported dependences of measured cerebral metabolite content on body composition (3-6). The initial studies (3-5) have reported for various populations, metabolites and brain regions that cerebral metabolite content was related to the body mass index (BMI) as a measure of body composition, suggesting a physiological connection between body composition and cerebral metabolite content. Specifically, negative correlations between NAA content or metabolite ratios were attributed to potential health related effects of obesity. While these studies thus interpreted this dependence as of a physiological nature, a large retrospective analysis of whole brain spectroscopic imaging data (6) did indeed confirm some dependence on BMl or body mass (which might be even stronger if other measures of obesity, like the waist-to-hip-ratio would be tested), but detailed analysis put the weight in the interpretation more on a potential technical nature since they had observed that the dependence on BMI largely went in parallel with a degradation of spectral quality. In particular, they found that the changes in metabolic concentrations and relative gray matter content were consistently associated with age, whereas their associations with BMl were inconsistent, and the associations of metabolite parameters with BMI were equally seen with body weight, thus suggesting that the metabolic variations were not associated with BMI per se. They also found a strong association of BMI or body weight with $\mathrm{B}_{0}$ inhomogeneity, spectral linewidth, SNR and error bounds, thus affecting the quality of spectroscopic quantification and contributing to the negative correlations of BMI with apparent metabolite content. 
Targeting a direct effect of lipid deposits near the investigated region of interest (ROI), Mon et al. (2) investigated whether superficial fat layers intentionally placed close to the spectroscopic $\mathrm{ROI}$ could influence the determined concentrations either expressed as metabolite ratios or when using unsuppressed water as referencing standard. Quite surprisingly, they found that in their settings the metabolite-to-water ratios were strongly reduced in the presence of superficial fat layers (10-35\% in phantoms, $16-24 \%$ in vivo), thus offering an alternative technical explanation to the observed correlations of metabolite content with $\mathrm{BMI}$, but also questioning the clinical use of water as a reference for quantification. Also, given the suspected frequency dependence of the effect (NAA reduced most, Cho least), these results questioned any simple means of absolute and relative quantification for MRS. However, this study had limitations, such as a small number of phantom and in vivo experiments, and in addition, it had not been performed using different acquisition settings to generalize the reported findings. Hence, in this study, we acquired single voxel spectroscopy data using a range of acquisition situations, like various localization and water suppression sequences, radio frequency (RF) coils, MR scanners, and two different field strengths to assess the conditions under which metabolite / water signal ratios are reduced in the presence of fat layers.

\section{METHODS:}

\section{Phantom preparation:}

All experiments were carried out in vitro using either experimental settings similar to those used by Mon et al. (2) or an experimental set up that takes the potential influence of local fat to the extreme. Fat layers were all composed of lard sold for cooking purposes and obtained at local stores. Where layers were needed, fat was filled into re-sealable plastic bags to form slabs of about $135 \times 95 \times 8 \mathrm{~mm}^{3}$.

Experiments were conducted on phantoms with different metabolite solutions, as illustrated in Fig. 1. A large spherical phantom, which will be referred to as P1 ("braino" from GE Medical systems, diameter of $16.9 \mathrm{~cm}$ ) containing $12.5 \mathrm{mM} \mathrm{N}$-acetylaspartate (NAA), $12.5 \mathrm{mM} \mathrm{L-}$ glutamic acid, $10 \mathrm{mM}$ creatine hydrate ( $\mathrm{Cr}$ ), $3 \mathrm{mM}$ choline chloride (Cho), $7.5 \mathrm{mM}$ myo-inositol, and, $5 \mathrm{mM}$ DL-lactic acid buffered in an aqueous phosphate buffer at near neutral $\mathrm{pH}$ ), a cylindrical phantom P2 (11.5 cm diameter; $20.5 \mathrm{~cm}$ length) containing $\sim 100 \mathrm{mM}$ acetate, $\sim 100$ $\mathrm{mM}$ glycine, and $\sim 100 \mathrm{mM}$ creatine in unbuffered solution, and two small spherical phantoms (5 $\mathrm{cm}$ diameter, same composition as P2) enclosed in cylindrical plastic bottles (11.5 cm diameter; 
$20.5 \mathrm{~cm}$ length) where for one (P3f) the bottle was filled with fat and for the other (P3w) it was filled with tap water as illustrated in Fig. 1. For P1 and P2 the effect of fat was studied by recording data in presence and absence of layers of fat placed on top of the phantoms.

\section{Data acquisition:}

The MR data was acquired on a 3-T (Trio, Siemens, Erlangen Germany) and a 7-T (Philips, Best, Netherlands) clinical whole body scanner either using phased array (PA) receive-only head coils with a body transmit coil $(3 T)$, a quadrature transmit-receive (TxRx) head coil (3 T), or a head transmit / 32-channel receive coil (7T). Each measurement session was started with basic MR imaging to define the setup and place ROIs for MRS. Afterwards $B_{1}$ at the ROI was determined by standard $B_{1}$ mapping techniques and the ROls were shimmed using first and second order $\mathrm{B}_{0}$ field gradients based on standard field mapping (Siemens' works in progress package "CVshim" at 3T) or a projection technique (Philips' higher order shimming tool "pencil beam volume shim" at 7T), where the methods used should at least in first order be able to cope with regions of fat because they were applied with echo time increments that make sure that the water and the main lipid signal at $1.3 \mathrm{ppm}$ are in-phase. Then, water-suppressed (WS) and unsuppressed (nWS) spectra were acquired, first with fat placed on the phantom (P1, P2) or phantom P3f, followed by equivalent and independent recordings without the fat layers or with phantom P3w. Pre-scan parameters, in particular shim and local $B_{1}$, were independently optimized for the ROls in each setting. Based on the type of scanner, RF coil and phantoms used, 5 different conditions were investigated as summarized in Table 1. The conditions were set up with a potentially increasing effect of fat. Condition 1) featured one fat layer only, while for conditions 2, 3, and 4 two fat layers were used, and finally the most extreme condition (5) was with a small test sample in a large fat or water enclosure. For conditions 1 to 4 , an ROI $(25 \times 25$ x $20 \mathrm{~mm}^{3}$ ) was placed $\sim 15 \mathrm{~mm}$ away (i.e. $15 \mathrm{~mm}$ gap between fat and ROI) from the fat layer(s) similar to the setup in (2). For condition 5, an ROI $\left(20 \times 20 \times 20 \mathrm{~mm}^{3}\right)$ was placed at the center of the small spherical phantom (inside the cylindrical bottle). Spectra were recorded with either PRESS or STEAM localization and with both long and short echo times (Table 1). In order to investigate the potential effect of radiation damping introducing nonlinear effects into the nWS reference scans as a consequence of manipulating the large water signal, some water suppressed scans were recorded with metabolite cycling (MC, parameters as used in Ref (7)) to subtract out the full-scale water signal compared to using standard water suppression with three presaturation pulses. 


\section{Data processing:}

MRS data were analyzed using jMRUI (8). The WS spectra were first eddy current-corrected using the phase information of the water reference (nWS) and then apodized using a Lorentzian function to match linewidths of the experiments with and without fat (see Fig. 2). Fitting was performed in jMRUI-QUEST (9) with base spectra modeled using VESPA (10) for the field strengths and echo times employed, but assuming ideal pulse sequences. The spectrum of $\mathrm{Cr}$ was split into two singlets with independent amplitude to account for effects of water suppression. The nWS water area was obtained by using either HSLVD (11) (1 component) or QUEST (9) with a singlet as basis set.

To juxtapose scans with and without fat, percent differences were calculated as (results with fat) minus (results without fat) relative to (results without fat).

\section{RESULTS:}

For the vast majority of measurements (59 out of 63), it was noted that linewidths were broader for the cases with fat than those without. The differences amounted to 0.01 to $2.2 \mathrm{~Hz}$ additional linewidth. Fig. 2 and Fig. 3 show some of the spectra for different conditions after adapting the linewidths and after scaling with the respective nWS water signals. Already visually, it can be appreciated that the peak amplitudes in all spectra from all the phantoms are very similar, independent of the presence or absence of fat. The detailed quantification results are summarized in Tables 2 to 4.

While the absolute signal areas ranged slightly but consistently higher for both water and metabolites with the superficial fat layer, the percentage differences of metabolite to water ratios were all small and within a 2 standard deviations (SD) range defined by the SD from repetition of measurements. They did not show a consistent trend for the different acquisition settings, phantoms or field strengths. For phantom P1 ("braino" with and without fat layers) the differences were below $5 \%$ in all cases and on average in magnitude below $2 \%$. Also for phantom P2, where we always used two fat layers and where the cylinder had a smaller diameter than the sphere in $\mathrm{P} 1$, the differences were even smaller $(<2 \%$ in all cases and on average in magnitude below $1 \%$ ).

For the final setup in phantoms P3f and P3w, where a small aqueous solution is totally immersed in either a large fat or water mass, the differences for the absolute signals were much bigger with $\sim 20 \%$ less signal for metabolites as well as water in the cases without fat, but with 
equally small differences for the metabolite to water ratios $(<5 \%$ in all cases, and on average below $2 \%$ in magnitude). The difference in absolute signals is related to coil loading, as reflected in the larger voltage needed to reach a $90^{\circ}$ flip angle with $\mathrm{P} 3 \mathrm{w}$ (87 V) compared to P3f $(116 \mathrm{~V})$. Correcting with the reciprocity principle $(12)$, the absolute signals remain constant within $3 \%$ on average.

\section{DISCUSSION}

Mon et al. (2) had reported an apparent systematic decrease in measured metabolite content in the presence of additional fat layers when metabolite tissue content was derived from water scaling. In our hands and with our settings, it was not possible to reproduce this effect. Contrary to their results, none of the various experimental settings that we tried showed this effect neither different field strengths and manufacturers, nor the use of transmit/receive coils or separate body transmit with local receive coils showed a consistent trend towards disproportional signal changes for water and metabolites or other frequency-dependence of the MR signals as a function of the presence of local fat depots. Similarly, there was no effect with or without water presaturation, and there was also no influence of localization sequence or echo time. We had also used different settings of fat layers vs. ROI in a spherical and cylindrical arrangement, and even going to the extreme of enclosing a small test sample in a large water or fat enclosure did not show any sign of the previously reported effect. However, we have not had the opportunity to exactly match the setting of Mon et al. with their specific hardware and a $4 \mathrm{~T}$ MR system. In addition, given that we could not pinpoint the effect in vitro, we did not proceed to investigate this effect in vivo, where Mon et al. (2) had reported similar effects as found in vitro. Still, we conclude that at least for a vast variety of experimental settings used in clinical MRS the presence of additional fat depots does not seem to jeopardize the use of water as an internal reference, and that metabolite ratios do not seem to depend on the amount of fat near the ROI.

In most of our experimental settings, we did observe larger field inhomogeneity when fat was placed near the ROI, either due to inherently larger $\mathrm{B}_{0}$ gradients or because of the inability of the shimming tools to handle large areas of fat when determining the local field map. This effect is reminiscent of the report by Maudsley et al. (6) who found in a retrospective analysis of whole brain chemical shift imaging data that larger linewidths were associated with increased BMI or patient weight - potentially representing situations with more fat depositions around or near the head. 
As expected, we also found some differences in absolute signal amplitudes, which could be explained by changes in coil loading leading to lower receive signals for larger loads; for the most extreme case with a large change in coil load (phantoms P3f and P3w investigated in a transmit/receive coil), signal correction based on the principle of reciprocity was efficient in eliminating these differences. For cases with non-identical transmit and receive coils (as used in Ref. (2)) the reciprocity principle cannot be applied to correct for changes in load. However, changes in coil load should not lead to the reported effects, if the transmit field is properly adjusted. If not, misadjusted flip angles in combination with off resonance terms could possibly lead to differential effects on water and metabolites because they could experience different effective transmit fields.

At this point, it is difficult for us to speculate on what circumstances led to the differences in signal intensity as reported by Mon et al., since not all potentially relevant details are listed in their report and - as stated in personal communication - neither their 4T scanner nor their head coil is operational anymore for further testing. Hence, the reasons for the discrepant findings of our studies remain speculative. If the current report rather speaks against a principle effect, like specific absorption of RF signals, as alluded to by Mon et al., we can only list a few factors that may in general lead to different relative signal strength between water and metabolites and also inbetween metabolites for acquisition settings with and without additional fat pools.

1) Changes in coil load with accompanying changes in $\mathrm{B}_{1}{ }^{+}$and $\mathrm{B}_{1}{ }^{-}$may be implicated as mentioned just above.

2) Changes in lineshape, as described above and probably caused by imperfect handling of the chemical shift effect of fat tissues by the shimming tools, can easily lead to wrong estimation of signal areas, and even though the lineshapes are identical for all peaks, including water, differently inaccurate fitting may occur for the situation of a dominant single water resonance and the case of a crowded metabolite spectrum with limited SNR.

3) A non-linear effect for water-suppressed and unsuppressed spectra can be caused by radiation damping, where the large water signal may feed back into the coil to produce an opposing RF field, which would not be present in the case of water presaturation. The size of this effect depends strongly on the type of RF coils used and is only expected to matter for coils with high $Q$ and proper tuning. The resonance width of the coil and its tuning depends on the coil load, which in turn may be influenced when adding superficial layers of fat. This was the background of why we tested water suppression without water presaturation. Even though we 
did not find an effect, specific RF coils (or possibly single coil elements) will show different susceptibility to this effect and care should definitely be used in applying water referencing in in vitro situations, where high $Q$ coils might cause the high signal situation to be very different from the low signal setting ${ }^{1}$.

4) Changes in coil combination efficiency for water suppressed and unsuppressed data may also lead to nonlinear intensities, but it is hard to see how this would also depend on coil load or fat layers, unless:

5) There might be direct signal bleed from surface fat layers in some coil elements, but that would be visible in the spectra.

In summary, the substantial in vitro and in vivo reduction in metabolite to water signal ratios and the frequency-dependent signal attenuation in the presence of additional fat depots reported in Ref. (2) for 4T could not be reproduced in vitro at 3T and 7T with any of the acquisition settings tested. From these results, it appears that water referencing is still a valid option under common acquisition conditions, but it remains to be evaluated what special factors lead to the observations reported in Ref. (2) and researchers may be well advised to investigate whether in their specific settings (small animal scanners, high resolution NMR, unusual RF coils or coil elements) radiation damping might complicate water referencing for quantification of MRS data.

\section{ACKNOWLEDGEMENTS}

This research was carried out in the framework of the European Marie-Curie Initial Training Network 'TRANSACT', PITN-GA-2012-316679, 2013-2017 and also supported by the Swiss National Science Foundation (\#320030_156952).

\footnotetext{
${ }^{1}$ Based on further technical information from the original $4 \mathrm{~T}$ head coil designer, Meyerhoff et al. (personal communication, June 2015) find it unlikely that radiation damping contributed significantly to the findings reported in Mon et al. (2) because the RF coil was judged to have too low a $Q$ to show relevant effects of radiation damping .
} 


\section{REFERENCES}

1. Oz G, Alger JR, Barker PB, Bartha R, Bizzi A, Boesch C, Bolan PJ, Brindle KM, Cudalbu C, Dincer A, Dydak U, Emir UE, Frahm J, Gilberto Gonzalez R, Gruber S, Gruetter R, Gupta RK, Heerschap A, Henning A, Hetherington HP, Howe FA, Huppi PS, Hurd RE, Kantarci K, Klomp D, Kreis R, Kruiskamp M, Leach M, Lin A, Luijten P, Marjańska M, Maudsley A, Meyerhoff D, Mountford C, Nelson S, Pamir M, Pan J, Peet A, Poptani H, Posse S, Pouwels P, Ratai E, Ross B, Scheenen T, Schuster C, Smith I, Soher B, Tkáč I, Vigneron D, Kauppinen $\mathrm{R}, \mathrm{MRS}$ Consensus Group. Clinical proton MR spectroscopy in central nervous system disorders. Radiology. 2014; 270: 658-679. Available at: doi:10.1148/radiol.13130531

2. Mon A, Abé C, Durazzo TC, Meyerhoff DJ. Effects of fat on MR-measured metabolite signal strengths: Implications for in vivo MRS studies of the human brain. NMR Biomed. 2013; 26: 1768-1774. Available at: doi:10.1002/nbm.3016

3. Gazdzinski S, Millin R, Kaiser LG, Durazzo TC, Mueller SG, Weiner MW, Meyerhoff DJ. BMI and neuronal integrity in healthy, cognitively normal elderly: a proton magnetic resonance spectroscopy study. Obesity (Silver Spring). Nature Publishing Group; 2010; 18 : 743-748. Available at: doi:10.1038/oby.2009.325

4. Gazdzinski S, Kornak J, Weiner MW, Meyerhoff DJ. Body mass index and magnetic resonance markers of brain integrity in adults. Ann Neurol. 2008; 63: 652-657. Available at: doi:10.1002/ana.21377

5. Gazdzinski S, Durazzo TC, Mon A, Meyerhoff DJ. Body mass index is associated with brain metabolite levels in alcohol dependence-a multimodal magnetic resonance study.

Alcohol Clin Exp Res. 2010; 34: 2089-2096. Available at: doi:10.1111/j.15300277.2010.01305.x

6. Maudsley AA, Govind V, Arheart KL. Associations of age, gender and body mass with $1 \mathrm{H}$ MR-observed brain metabolites and tissue distributions. NMR Biomed. 2012; 25: 580593. Available at: doi:10.1002/nbm.1775

7. MacMillan EL, Chong DGQ, Dreher W, Henning A, Boesch C, Kreis R. Magnetization exchange with water and T1 relaxation of the downfield resonances in human brain spectra at 3.0 T. Magn Reson Med. 2011; 65: 1239-1246. Available at: doi:10.1002/mrm.22813

8. Stefan D, Cesare F Di, Andrasescu A, Popa E, Lazariev A, Vescovo E, Strbak O, Williams S, Starcuk Z, Cabanas M, van Ormondt D, Graveron-Demilly D. Quantitation of magnetic resonance spectroscopy signals: the jMRUI software package. Meas Sci Technol. 2009; 20: 104035(9pp). Available at: doi:10.1088/0957-0233/20/10/104035 
NMR in Biomedicine - For Peer Review Only

Page 12 of 38

9. Ratiney H, Coenradie Y, Cavassila S, Van Ormondt D, Graveron-Demilly D. Time-domain quantitation of $1 \mathrm{H}$ short echo-time signals: Background accommodation. Mage Meson Mater Shy, 2004; 16: 284-296. Available at: doi:10.1007/s10334-004-0037-9

10. Sober B, Semanchuk D, Todd D, Steinberg J, Young K. Vespa: Integrated applications for RF pulse design, spectral simulation and MRS data analysis. Proceedings of the 19th Annual Meeting ISMRM, Montreal,Canada,2011; 1410.

11. Laudadio T, Mastronardi N, Vanhamme L, Hecke P Van, Huffel S Van. Improved Lanczos Algorithms for Blackbox MRS Data Quantitation. J Magn Reson. 2002; 157: 292-297. Available at: doi:10.1006/jmre.2002.2593

12. Hoult DI. The NMR receiver: A description and analysis of design. Prog NMR Spectrosc. 1978; 12: 41-77. Available at: doi:10.1016/0079-6565(78)80002-8.

12

http://mc.manuscriptcentral.com/nbm 


\section{Figure Captions:}

\section{Figure 1:}

MR images illustrating the experimental setups for different phantoms (a) spherical phantom (P1), (b) cylindrical phantom (P2), (c) small spherical phantom enclosed in a bottle (P3f and $\mathrm{P} 3 \mathrm{w}$ ) containing either fat or water, where the images were recorded with and without fat saturation to highlight the difference.

\section{Figure 2:}

Linewidth-matched spectra (scaled by unsuppressed water) acquired using different acquisition conditions in the presence (blue) and absence (green) of superficial fat layers on phantom P1.

\section{Figure 3:}

Linewidth-matched spectra (scaled by unsuppressed water) acquired using different acquisition conditions in the presence (blue) and absence (green) of superficial fat layers for phantom P2 and for phantoms P3f (blue) vs. P3w (green), respectively. 


\section{TABLES}

Table 1: Acquisition settings for all experimental conditions

\begin{tabular}{|c|c|c|c|c|c|c|c|c|c|}
\hline condition & $\mathrm{B}_{0}$ & phantom & $\begin{array}{l}\text { \# of fat } \\
\text { layers }\end{array}$ & $\begin{array}{c}\text { head } \\
\text { coil }\end{array}$ & $\begin{array}{c}\text { no. of } \\
\text { sessions }\end{array}$ & $\begin{array}{l}\text { voxel } \\
\text { size }\end{array}$ & sequence & $\begin{array}{l}\mathrm{TE} \\
\text { (ms) }\end{array}$ & $\begin{array}{c}\text { TR } \\
\text { (ms) }\end{array}$ \\
\hline \multirow{3}{*}{1} & \multirow{3}{*}{ 3T } & \multirow{3}{*}{ P1 } & \multirow{3}{*}{1} & \multirow{3}{*}{ PA } & \multirow{3}{*}{5} & \multirow{3}{*}{$\begin{array}{l}12.5 \\
\mathrm{~cm}^{3}\end{array}$} & PRESS & 80 & 2000 \\
\hline & & & & & & & STEAM & 20 & 2000 \\
\hline & & & & & & & STEAM & 80 & 2000 \\
\hline \multirow{3}{*}{2} & \multirow{3}{*}{ 3T } & \multirow{3}{*}{ P1 } & \multirow{3}{*}{2} & \multirow{3}{*}{ TxRx } & \multirow{3}{*}{5} & \multirow{3}{*}{$\begin{array}{l}12.5 \\
\mathrm{~cm}^{3}\end{array}$} & PRESS & 80 & 2000 \\
\hline & & & & & & & PRESS-MC* & 80 & 2000 \\
\hline & & & & & & & PRESS-MC+WS & 80 & 2000 \\
\hline \multirow{2}{*}{3} & \multirow{2}{*}{$7 \mathrm{~T}$} & \multirow{2}{*}{ P1 } & \multirow{2}{*}{2} & \multirow{2}{*}{ PA } & \multirow{2}{*}{3} & \multirow{2}{*}{$\begin{array}{l}12.5 \\
\mathrm{~cm}^{3}\end{array}$} & STEAM & 20 & 2000 \\
\hline & & & & & & & STEAM & 80 & 2000 \\
\hline \multirow{3}{*}{4} & \multirow{3}{*}{ 3T } & \multirow{3}{*}{ P2 } & \multirow{3}{*}{2} & \multirow{3}{*}{ TxRx } & \multirow{3}{*}{6} & \multirow{3}{*}{$\begin{array}{l}12.5 \\
\mathrm{~cm}^{3}\end{array}$} & STEAM & 20 & 5000 \\
\hline & & & & & & & STEAM & 80 & 5000 \\
\hline & & & & & & & PRESS & 80 & 5000 \\
\hline \multirow{3}{*}{5} & \multirow{3}{*}{ 3T } & \multirow{3}{*}{$\begin{array}{l}\text { P3f and } \\
\text { P3w }\end{array}$} & \multirow{3}{*}{$\begin{array}{c}\text { Fat } \\
\text { enclosure }\end{array}$} & \multirow{3}{*}{ TxRx } & \multirow{3}{*}{3} & \multirow{3}{*}{$8 \mathrm{~cm}^{3}$} & STEAM & 20 & 5000 \\
\hline & & & & & & & STEAM & 80 & 5000 \\
\hline & & & & & & & PRESS & 80 & 5000 \\
\hline
\end{tabular}

${ }^{*} \mathrm{MC} \rightarrow$ metabolite-cycling for water suppression (WS)

${ }^{\#} \mathrm{MC}+\mathrm{WS} \rightarrow \mathrm{MC}$ plus standard WS 
Table 2: Quantification results for different acquisition settings using phantom $P 1$

\begin{tabular}{|c|c|c|c|c|c|c|c|c|c|c|c|c|}
\hline \multirow[t]{2}{*}{$\begin{array}{c}\text { conditio } \\
n\end{array}$} & \multirow[t]{2}{*}{$B_{0}$} & \multirow[t]{2}{*}{ coil } & \multirow[t]{2}{*}{$\begin{array}{c}\text { se- } \\
\text { quence }\end{array}$} & \multirow[t]{2}{*}{$\begin{array}{c}\text { \# of } \\
\text { spectra }\end{array}$} & \multirow[t]{2}{*}{$\begin{array}{c}\mathrm{TE} \\
{[\mathrm{ms}]}\end{array}$} & \multicolumn{4}{|c|}{$\begin{array}{c}\text { \% difference } \\
\text { for absolute values } \\
\text { mean (SD) }\end{array}$} & \multicolumn{3}{|c|}{$\begin{array}{l}\% \text { difference } \\
\text { for ratios to water } \\
\text { mean (SD) }\end{array}$} \\
\hline & & & & & & NAA & Cho & $\mathrm{Cr}$ & $\mathrm{H}_{2} \mathrm{O}$ & NAA & Cho & $\mathrm{Cr}$ \\
\hline 1 & $3 \mathrm{~T}$ & PA & $\begin{array}{c}\text { PRESS } \\
\text { MC }\end{array}$ & 5 & 80 & $\begin{array}{c}5.0 \\
(2.9)\end{array}$ & $\begin{array}{c}0.7 \\
(5.9)\end{array}$ & $\begin{array}{c}0.9 \\
(3.1)\end{array}$ & \begin{tabular}{|c|}
1.4 \\
$(0.4)$
\end{tabular} & $\begin{array}{c}3.5 \\
(3.3)\end{array}$ & $\begin{array}{l}-0.7 \\
(6.2)\end{array}$ & $\begin{array}{c}-0.5 \\
(3.4)\end{array}$ \\
\hline 1 & $3 \mathrm{~T}$ & PA & $\begin{array}{l}\text { PRESS } \\
\text { MC+WS }\end{array}$ & 5 & 80 & $\begin{array}{c}3.3 \\
(1.8)\end{array}$ & $\begin{array}{l}-0.7 \\
(1.2)\end{array}$ & $\begin{array}{c}1.7 \\
(2.5)\end{array}$ & $\begin{array}{c}1.1 \\
(0.8)\end{array}$ & $\begin{array}{c}2.2 \\
(1.3)\end{array}$ & $\begin{array}{l}-1.7 \\
(1.1)\end{array}$ & $\begin{array}{c}0.7 \\
(1.7)\end{array}$ \\
\hline 1 & $3 \mathrm{~T}$ & PA & $\begin{array}{l}\text { PRESS } \\
\text { WS }\end{array}$ & 5 & 80 & $\begin{array}{c}4.3 \\
(2.3)\end{array}$ & $\begin{array}{c}2.6 \\
(5.1)\end{array}$ & $\begin{array}{c}4.9 \\
(4.8)\end{array}$ & $\begin{array}{c}1.9 \\
(0.5)\end{array}$ & $\begin{array}{c}2.4 \\
(1.9)\end{array}$ & $\begin{array}{c}0.7 \\
(4.6)\end{array}$ & $\begin{array}{c}3.0 \\
(4.3)\end{array}$ \\
\hline 2 & $3 T$ & Tx-Rx & $\begin{array}{l}\text { STEAM } \\
\text { WS }\end{array}$ & 5 & 20 & $\begin{array}{c}2.9 \\
(3.9)\end{array}$ & $\begin{array}{c}7.0 \\
(9.6)\end{array}$ & $\begin{array}{c}2.4 \\
(2.4)\end{array}$ & $\begin{array}{c}2.3 \\
(1.5)\end{array}$ & $\begin{array}{c}0.5 \\
(2.5)\end{array}$ & $\begin{array}{c}4.5 \\
(8.0)\end{array}$ & $\begin{array}{c}0.1 \\
(1.4)\end{array}$ \\
\hline 2 & $3 \mathrm{~T}$ & $T x-R x$ & $\begin{array}{c}\text { STEAM } \\
\text { WS }\end{array}$ & 5 & 80 & $\begin{array}{c}1.3 \\
(3.5)\end{array}$ & $\begin{array}{c}2.3 \\
(14.2)\end{array}$ & $\begin{array}{c}1.2 \\
(8.0)\end{array}$ & $\begin{array}{c}2.1 \\
(1.6)\end{array}$ & $\begin{array}{l}-0.7 \\
(3.0)\end{array}$ & $\begin{array}{c}0.2 \\
(13.2)\end{array}$ & $\begin{array}{c}-0.9 \\
(7.3)\end{array}$ \\
\hline 2 & $3 T$ & $T x-R x$ & $\begin{array}{l}\text { PRESS } \\
\text { WS }\end{array}$ & 5 & 80 & $\begin{array}{c}4.5 \\
(2.3)\end{array}$ & $\begin{array}{c}2.4 \\
(5.6)\end{array}$ & $\begin{array}{c}4.2 \\
(5.1)\end{array}$ & $\begin{array}{c}2.3 \\
(2.0)\end{array}$ & $\begin{array}{c}2.2 \\
(1.6)\end{array}$ & $\begin{array}{c}0.1 \\
(5.0)\end{array}$ & $\begin{array}{c}1.7 \\
(4.3)\end{array}$ \\
\hline 3 & $7 \mathrm{~T}$ & PA & $\begin{array}{c}\text { STEAM } \\
\text { WS }\end{array}$ & 3 & 20 & $\begin{array}{c}1.7 \\
(1.9)\end{array}$ & $\begin{array}{c}7.0 \\
(5.6)\end{array}$ & $\begin{array}{c}0.9 \\
(3.3)\end{array}$ & $\begin{array}{c}5.1 \\
(3.4)\end{array}$ & $\begin{array}{l}-3.3 \\
(1.7)\end{array}$ & $\begin{array}{c}1.7 \\
(2.1)\end{array}$ & $\begin{array}{l}-3.9 \\
(0.3)\end{array}$ \\
\hline 3 & $7 \mathrm{~T}$ & PA & $\begin{array}{c}\text { STEAM } \\
\text { WS }\end{array}$ & 3 & 80 & $\begin{array}{c}6.3 \\
(1.9)\end{array}$ & $\begin{array}{c}9.4 \\
(0.3)\end{array}$ & $\begin{array}{c}6.3 \\
(2.6)\end{array}$ & $\begin{array}{c}8.2 \\
(1.9)\end{array}$ & $\begin{array}{l}-1.7 \\
(1.9)\end{array}$ & $\begin{array}{c}1.2 \\
(1.9)\end{array}$ & $\begin{array}{l}-1.7 \\
(3.1)\end{array}$ \\
\hline
\end{tabular}


NMR in Biomedicine - For Peer Review Only

Page 16 of 38

1
2
2
3
4
5
6
7
7
8
9
10
11
12
13
14
15
15
16
17
18
19
20
21
22
23
23
24
25
26

Table 3: Quantification results for different acquisition settings using phantom P2

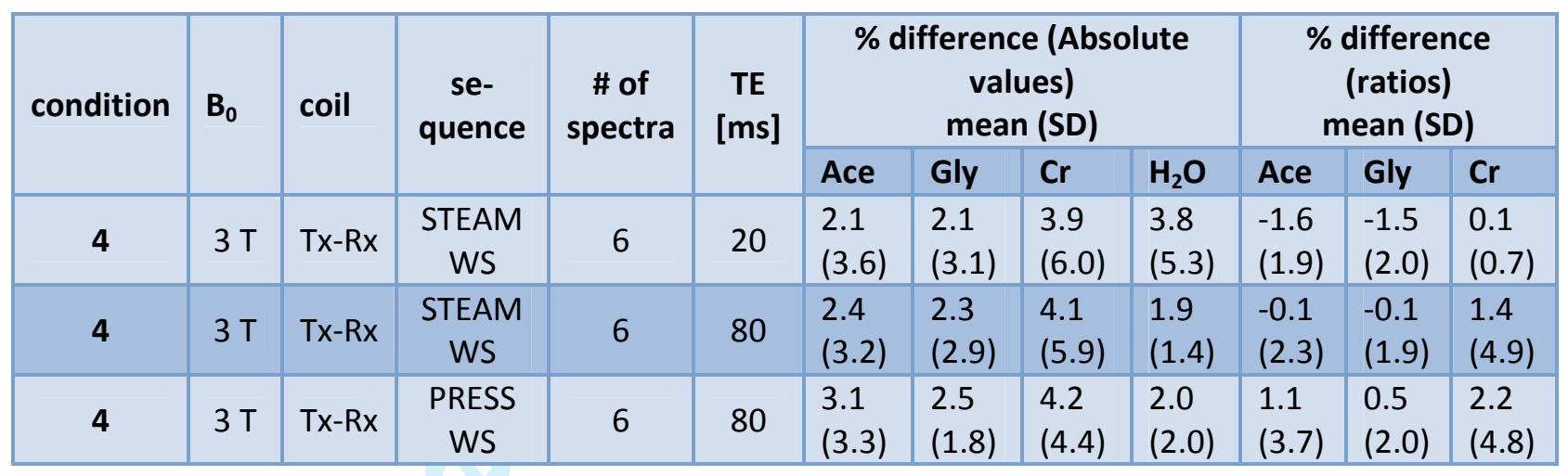

16

http://mc.manuscriptcentral.com/nbm 

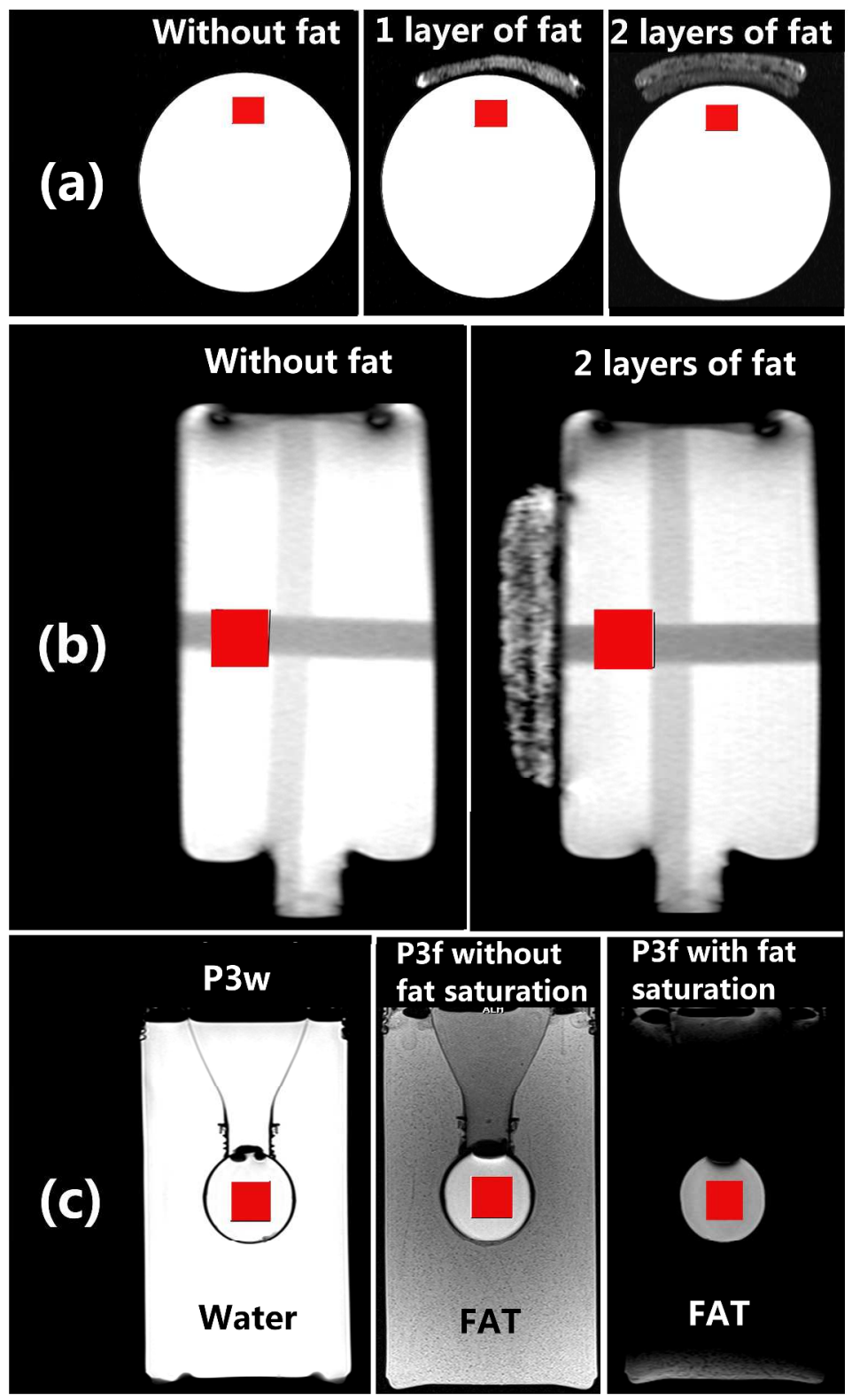

Figure 1:

MR images illustrating the experimental setups for different phantoms (a) spherical phantom (P1), (b) cylindrical phantom (P2), (c) small spherical phantom enclosed in a bottle (P3f and P3w) containing either fat or water, where the images were recorded with and without fat saturation to highlight the difference.

$$
320 \times 528 \mathrm{~mm}(300 \times 300 \text { DPI })
$$




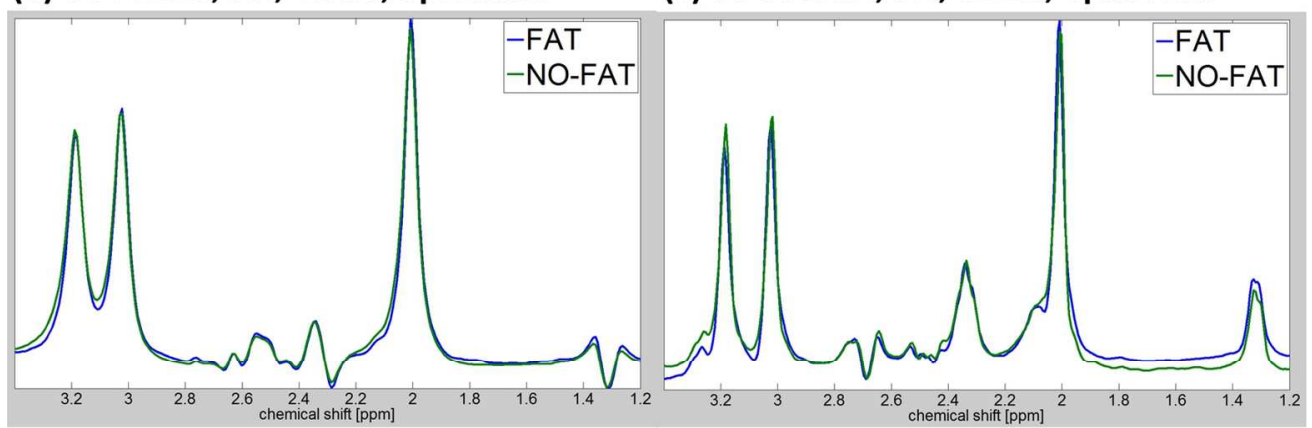

(b) 3T PRESS, TxRx coil, TE80, Spherical (d) 7T STEAM, PA coil, TE80 Spherical

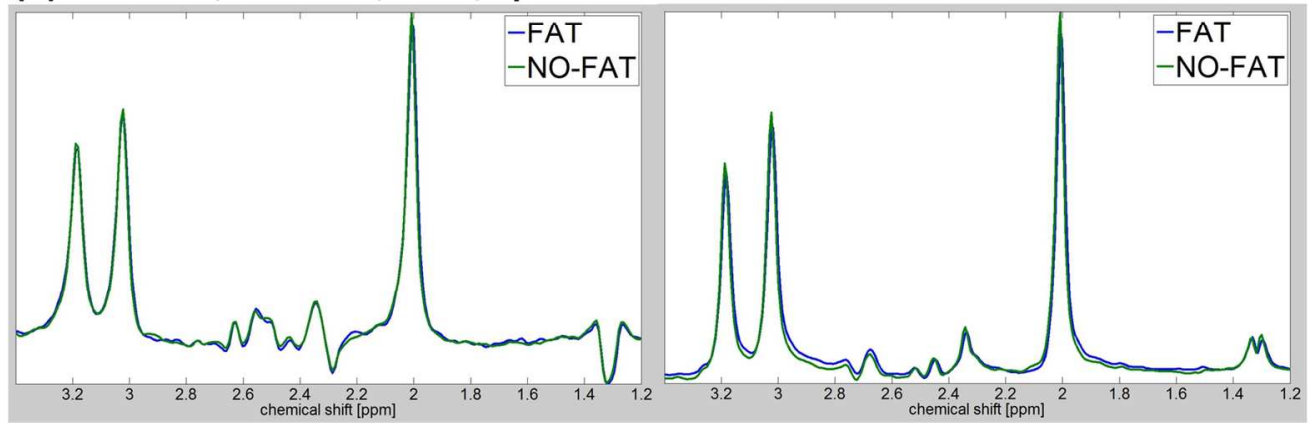

Figure 2:

Linewidth-matched spectra (scaled by unsuppressed water) acquired using different acquisition conditions in the presence (blue) and absence (green) of superficial fat layers on phantom P1.

$$
142 \times 101 \mathrm{~mm}(300 \times 300 \text { DPI) }
$$




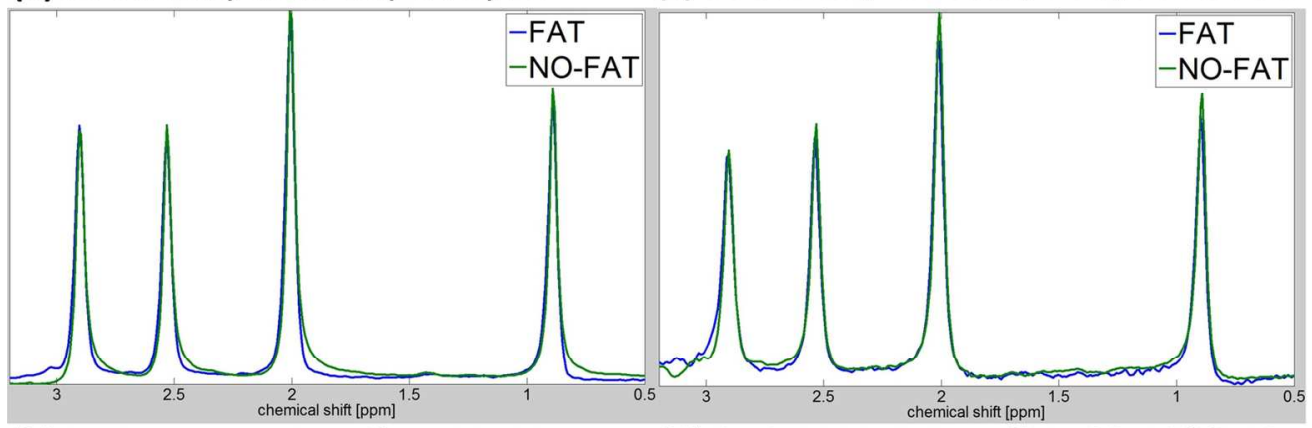

(b) 3T STEAM, TxRx coil, TE80, P2

(d) 3T STEAM, TxRx coil, TE80, P3f \& P3W

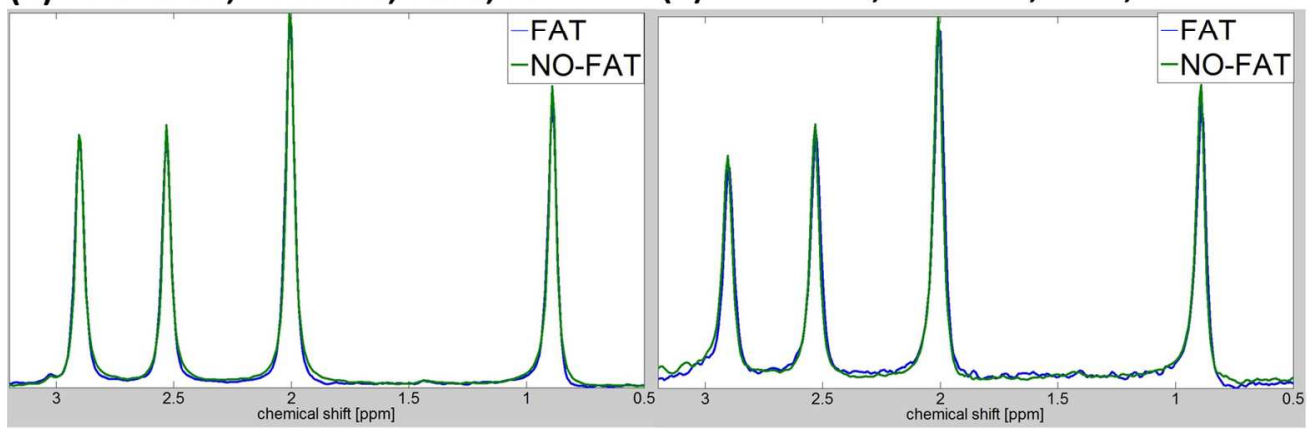

Figure 3:

Linewidth-matched spectra (scaled by unsuppressed water) acquired using different acquisition conditions in the presence (blue) and absence (green) of superficial fat layers for phantom P2 and for phantoms P3f (blue) vs. P3w (green), respectively.

$142 \times 101 \mathrm{~mm}(300 \times 300$ DPI $)$ 


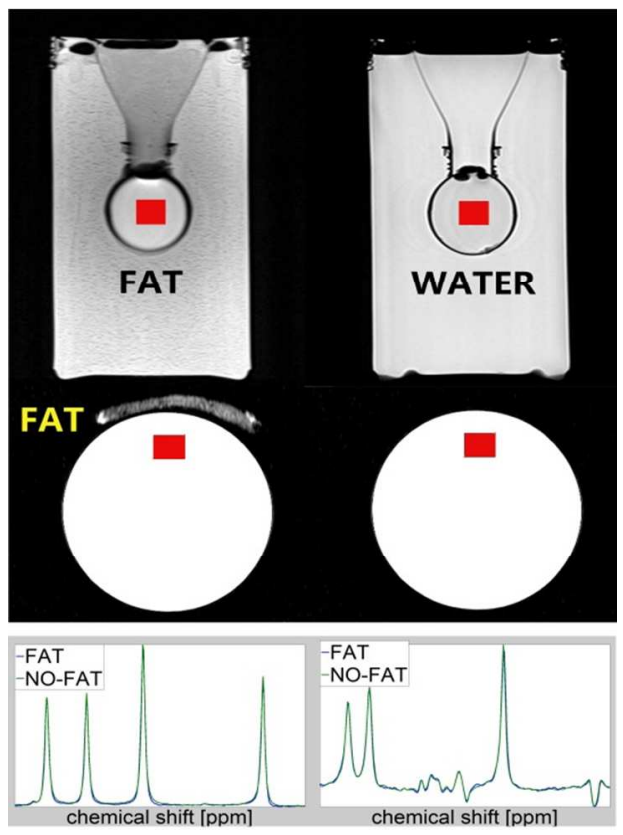

Does superficial fat affect metabolite concentrations determined by MR spectroscopy with water referencing?

S.P. Kyathanahally, N.D. Fichtner, V. Adalid, R. Kreis

A recent ${ }^{1} \mathrm{H}$ MR spectroscopy study reported frequency dependent signal attenuation in the presence of superficial fat layers, thus questioning the clinical use of water as a reference for quantification and quantitative spectroscopy as a whole. We have attempted to verify this effect and pinpoint the potential causes by acquiring data with various acquisition settings. With all the settings the reported effects could not be reproduced. We thus conclude that water referencing remains a valid option under common acquisition conditions. 